\title{
Le tournant substantialiste de la laïcité française
}

\section{The substantivist turn of French laicism}

Philippe Portier ${ }^{\star}$

*École Pratique des Hautes Études - Paris, France

philippe.portier@gsrl.cnrs.fr 


\title{
Résumé
}

La laïcité française a connu un tournant au cours des trente dernières années. La loi du 9 décembre 1905 avait établi une laïcité libérale. Ce modèle comportait une double face. D'abord, il établissait une séparation entre l'Etat et les cultes: le pouvoir ne pouvait désormais ni les reconnaître, ni les subventionner. Ensuite, il accordait aux religions une complète liberté d'organisation et de communication. Or, au cours de ces dernières décennies, la laïcité s'est reconfigurée. L'Etat a établi, d'une part, des dispositifs de reconnaissance inédits sur le terrain financier et symbolique; il a, d'autre part, introduit des mesures inédites de surveillance des cultes et de neutralisation de l'expression religieuse. On parle ici d'un «tournant substantialiste»: le gouvernement semble bien en effet vouloir s'ériger en garant d'une éthique sociale lourde, correspondant à ce que certains acteurs politiques et certains intellectuels nomment, depuis les années 1980, l' «identité française». Cette contribution entend analyser cette mutation en s'arrêtant sur l'enchaînement de trois phénomènes: la transformation des paysages religieux, l'évolution des discours politiques, la transformation des règles juridiques.

Mots-clés: laïcité; polarisation religieuse; liberté religieuse; républicanisme.

\begin{abstract}
The French secularism knew a dramatic change during the last thirty years. The law of December 9th, 1905 had established a liberal secularism. This model contained a double face. Firstly, it established a separation between the State and the churches: the Government could not either recognize them, or subsidize them. Secondly, it granted to the religions a complete freedom of organization and communication. During these last decades, the secularism recomposed itself. The State established, on one hand, new devices of financial and symbolic recognition; it established, on the other hand, new measures of control of the churches and attempted to neutralize religious expressions in the public sphere. We speak here of a "substantialist turning point": the Government seems indeed to set up itself as the promoter of a heavy social ethics, related to what some political leaders call the "French identity". This contribution intends to analyze this transformation by exploring three phenomena: the transformation of the religious landscapes, the evolution of the political speeches, the reshaping of the legal rules.
\end{abstract}

Keywords: secularism; polarization; freedom of religion; republicanism. 


\section{Introduction $^{1}$}

Hegel (1998, p. 201), dans ses Principes de philosophie du droit, décrivait ainsi la mutation politique engagée, en Occident, aux XVII-XVIIIe siècles: «L'époque est celle de l'esprit libre. Le droit de la liberté subjective constitue le point critique et central de la différence entre l'Antiquité et les temps modernes. [...] Ce droit a été fait principe effectif d'une nouvelle forme du monde.»

Cette «nouvelle forme du monde» articule deux éléments clés, étroitement corrélés. Elle se constitue, d'abord, sur l'assise d'une recomposition de l'idée du sujet. On pensait l'existence humaine, hier, à partir du paradigme de la dépendance: l'homme se trouvait rivé à la normativité que Dieu avait établie. La liberté n'avait rien alors d'une auto-affirmation de soi. Elle trouvait sa signification dans le fait de se soumettre aux "prescriptions de la loi éternelle». La rupture moderne fait prévaloir une axiomatique de l'autonomie: tandis que se défait l'ordre finalisé de la nature, le sujet devient alors à lui-même son propre centre. La philosophie des droits de l'homme exprime juridiquement ce tournant: chacun se voit reconnaître des facultés premières (liberté de croyance et liberté d'expression, droit de propriété et liberté du travail notamment), à partir desquels il pourra construire son propre itinéraire d'existence. L'ordre moderne s'affirme, ensuite, sur l'assise d'une reconfiguration de l'idée du pouvoir. Le gouvernement s'inscrivait, dans lâge ancien des choses, dans la "grande chaîne de l'être»: issu de la volonté de Dieu, il avait mission de conduire ses sujets sur le chemin du bien et du salut. Rien de cette politique de la vérité ne demeure dans la civilisation nouvelle. Tout se passe désormais «comme si Dieu n'existait pas", etsi Deus non daretur, selon la formule de Grotius. Laloi de l'Etat, loin de toute référence à la bona vita, ne se donne plus pour viséeque de poserles conditions de possibilité d'un exercice harmonieux des droits de l'homme. Elle n'unifie plus les pensées et les comportements; elle se satisfait simplement d’ordonner les innombrables pluralismes émanés de la société civile.

Cette immanentisation de l'ordre social laisse une question en suspens: quel statut accorder la figure divine, et aux Eglises qui en administrent

1 La version initiale de ce texte a été présentée lors du colloque inaugural du Centre européen des études républicaines en novembre 2016. Le colloque est appelé à être publié aux éditions du Bord de l'Eau. 
le culte, dans cet univers ainsi livré à ses propres raisons? Les gouvernements modernes ont tenté de résoudre le problème en inventant la «solution laïque». L'expression décrit un régime de droit, marqué, d'une part, par le respect de la liberté de conscience, d'autre part, par l'affirmation de la neutralité de l'Etat. Les formes concrètes de ce dispositif dépendent de la signification attribuée, ex loco et ex tempore, aux deux notions structurantes - liberté et neutralité - à l'instant signalées. En France, depuis la sortie de la décennie révolutionnaire, deux formes de sécularité se sont succédé.Au XIXe siècle, c'est le système concordataire qui s'impose: il octroie un statut officielaux cultes catholique, réformé, luthérien, juif - ce qui leur vaut subventionnement -, tout en les soumettant à la surveillance de l'administration. Si le politique et le religieux s'enchevêtrent, ce n'est cependant pas en remettant en cause les principes de la modernité: la liberté publique de conscience est ici globalement garantie dans le cadre d'un Etat qui, loin de s'ordonner à la loi numineuse, affirme puissamment sa souveraineté. La Troisième République invente un autre modèle, séparatiste celui-là. Préparé dès les années 1880 , il trouve sa forme aboutie dans la loi de séparation des Eglises et de l'Etat du 9 décembre 1905. D'un côté, il privatise le religieux: exclus de la sphère d'étaticité, les groupements confessionnels se trouvent constitués en institutions de droit privé, laissées d'ailleurs à leur autonomie de fonctionnement. De l'autre, il universalise le politique: refusant toute expression en leur sein de la particularité religieuse, les institutions étatiques s'exhaussent au niveau de la raison commune. Il s'agit, expliquent les républicains, de placer la communauté politique, et les citoyens qui la constituent, en dehors de toute allégeance à l'autorité extérieure du religieux, et notamment de l'Eglise catholique alors rivée à une intransigeance toute «syllabique et vaticanesque ${ }^{2}$

Or, ce modèle de laïcité s'est trouvé confronté, à partir des années 1970-1980, à l'expression de revendications identitaires: leurs promoteurs se sont attachés, de manière assez inédite, à faire valoir, jusque dans l' «espace public ", leur identité religieuse. Comment l'Etat, tenu par le modèle d'égalité abstraite de la loi de 1905 , a-t-il réagi ? On voudrait montrer ici qu'il s'est, au cours de ces dernières années, engagé dans une transformation décisive de son système de régulation

2 Sur ces points, Philippe Portier (2016a). 
du religieux: celui-ci s'agençait hier autour du principe de séparation de l'institution politique et de l'institution religieuse; il se construit désormais selon un dispositif, tout à la fois recognitif et perfectionniste, d'intrication des deux instances. Cette reconfiguration - où se révèle le passage d'un «régime de la norme " à un "régime de la valeur ", pour parler comme Jürgen Habermas (1992) est le fruit d'une situation marqué par un déplacement des enjeux, un renouvellement des discours, une transformation des règles.

\section{Le déplacement des enjeux}

Dans les années 1960-1970, la laïcité ne faisait plus réellement question, sauf, à titre résiduel, lorsqu'on évoquait la question de l'école. On plaçait alors l'« hypothèse socialiste» au centre de la délibération collective. Cette élision était due au fait que l'objet à partir duquel elle s'était pensée semblait en voie d'épuisement. La sociologie, celle des religions avec Bryan Wilson comme celle du politique avec Gabriel Almond, annonçait sa mort imminente: le religieux était voué, dans le monde de la "culture civique», à s'effacer de notre champ d'expérience publique, et même de notre horizon d'attente privé. C'était le temps où triomphait le paradigme de la sécularisation, par lequel on pensait l'effacement de la croyance comme un phénomène irréversible et homogène. Or, au cours de ces quatre dernières décennies, la donne s'est transformée. Nous nous sommes trouvés, un peu partout en Europe, travaillés de nouveau par la question religieuse. Comment caractériser ce nouvel état de la société? Le paradigme de la désécularisation, développé par Peter Berger (1999) (qui fut, en son temps, l'un des tenants de la théorie conventionnelle de la sécularisation), est probablement trop univoque. Il faut lui préférer celui de la polarisation, défendu par James Campbell (2016) notamment. L'expression veut saisir deux tendances antinomiques dans les sociétés occidentales, et, en leur sein, dans la société française.

Ce qui marque, c'est, certes, la dissolution des appartenances religieuses: les populations persistent à vouloir se séparer de Dieu. On le voit au niveau des affiliations pratiques. D'année en année, comme le montrent les diverses enquêtes, le nombre des sans religion augmente. Dans les années 1950, on compte, mesurés par les statistiques sur le baptême et par les premiers 
sondages d'opinion, $92 \%$ de catholiques déclarés; et à peine $5 \%$ de sans religion. En 2008, les catholiques déclarés sont $42 \%$ dans la population globale, seulement $23 \%$ dans la génération des $18-29$ ans. $8 \%$ des Français, à peine, sont des pratiquants réguliers; $10 \%$ des Français relèvent des autres dénominations (juive, protestante, musulmane, bouddhiste...). Près de la moitié de la population se place dans la catégorie des sans religion. ${ }^{3}$ L'examen des adhésions normatives est plus significatif encore. La diminution des affiliations s'est accompagnée d'une récusation des croyances et des principes portés par les institutions ecclésiales. Le phénomène touche au premier chef, bien sûr, la catégorie des sans religion. Souvent, ces derniers sont plus intéressés par les questions de spiritualité que leurs homologues des périodes antérieures (Willaime, 2007). Il leur arrive même de cultiver, parfois, la mémoire des «racines chrétiennes» de la France. Ils sont cependant fort éloignés des dogmes chrétiens, et, sur le terrain des options morales, plus libérauxque les Français qui se placent du côté du pôle religieux. La population affiliée constitue-t-elle un bloc homogène de fidélité? Aucunement. L'observation la révèle, même si c'est à moindre titre que la population des sans religion, affectée par de lourds processus de "sécularisation interne", que signalent la subjectivation de son rapport aux contenus de foi, et, souvent, son adhésion au libéralisme culturel. A propos de l'avortement par exemple, les catholiques pratiquants constituent certes, en France, le groupe social le moins permissif en la matière; il reste qu'une majorité d'entre eux (53\%) se dit favorable à un droit sans restriction à l'IVG, alors que ce n'était le cas que de $37 \%$ de la même catégorie en $1974 .{ }^{4}$

Mais la sécularisation n'est pas le tout de ce moment de l'histoire. On assiste aussi, depuis une quarantaine d’années, à une certaine réaffirmation des identités confessionnelles. Aucun des mondes religieux ne se tient à l'écart de cette "revanche de Dieu », ni le monde juifqui, au cours de ces dernières années, a vu se consolider ses familles orthodoxes et surtout ultra-orthodoxes, ni le monde protestantau sein duquel le courant évangélique a connu une expansion impressionnante, ni le monde catholique, comme on l'a vu, par exemple, lors de

3 On s'appuie ici sur les enquêtes sur les Valeurs des Européens (European Values Surveys) en 1981, 1990, 1999 et en 2008. Y sont posées une trentaine de questions sur les attitudes religieuses (Bréchon; Gonthier, 2014).

4 Sondages IFOP, 1974-2014. Analyse comparative dans Atlantico (1972-2014: comment..., 2014). 
la controverse autour du «mariage pour tous». La même tendance marque l'univers musulman, et de manière bien plus visible socialement,parce que la population musulmane est numériquement importante, parce que l'affirmation de son appartenance tranche davantage avec la culture d'une société demeurée attachée à tout un corpus de gestes chrétiens, parce que demeure enfin dans le tréfonds de la société française une mémoire de l'antagonisme colonial. Ce revival musulman, dont on voit les indices dès la fin des années 1980, en particulier dans les jeunes générations mal intégrées (ou qui s'estiment discriminées5), s'exprime souvent dans le cadre de la sphère privée, où se sont développés la pratique de la prière, l'observance du ramadan, le respect des obligations alimentaires (autour du halal). Il affecte aussi la sphère publique: l'affirmation de l'identité s'accompagne souvent, en effet, d'une demande de reconnaissance adressée aux pouvoirs publics. Cette demandevaut sur le terrain financier (aide à la construction de mosquées), sur le terrain politique (insertion dans les dispositifs de participation politique), sur le terrain symbolique (obtention du droit de porter des signes religieux dans l'espace étatique). C'est d'ailleurs, principalement, autour de cette question-là, celle du vêtement, si prégnante en France depuis la Révolution de 1789, que s'est nouée la dispute publique. Olivier Roy (2005, p. 105) résumait ainsi cette évolution de la population musulman:

L'immigré des années 1970 était silencieux: on parlait pour lui. Les jeunes beurs des années 1980, quand ils sortaient de leurs banlieues, revendiquaient le discours dominant sur l'intégration, au lieu de défendre une différence, sinon dans la couleur de peau [...]. Ce qui est apparu plus tard, dans les années 1990, c'est un discours islamique structuré [...] qui fait [...] l'apologie d'une différence fondamentale, celle d'une croyance qui s'exhibe sans complexe.

Ces requêtes de reconnaissance publiqueauraient pu ne pas provoquer le débat. Il n'en a pas été ainsi : sans que soient négligés les enjeux relatifs au traitement du chômage ou au devenir de l'Europe, le thème de la religion est redevenu central au cours de ces dernières années, à partir désormais d'un questionnement, non plus, sauf marginalement, sur la place du catholicisme dans l'espace

5 Voir à ce sujet l'enquête d'Olivier Galland et Anne Muxel (2017). 
public, mais sur la «compatibilité de l'islam avec les valeurs de la République». Malgré quelques signes avant-coureurs, le débat émerge clairement à partir de l'affaire des foulards du collège de Creil en 1989, dans un contexte échauffé par l'affaire des Versets sataniques de Salman Rushdie. Trois raisons peuvent expliquer ce retour. Une raison politique: la chute du mur de Berlin, la disparition du Parti communiste, la libéralisationdoctrinale du Parti socialiste ont installé la société française dansune situation «postsocialiste» qui a libéré un espace de discursivité à l'intérieur duquel ont pu s'affirmer de nouveaux objets de controverse. Une raison culturelle: la France républicaine s'est construite sur le fondement d'une culture de la généralité, qui lui fait ressentir comme un troublel'expression publique des signes d'appartenance particulière, surtout lorsqu'elle concerne une religion avec laquelle tous les contentieux n'ont pas été réglés. Une raison sociale enfin: puissante dans les années 1970, l'idée de la diversité heureuse, fondée sur l'harmonie naturelle des différences, s'est trouvée frappée de décroyance, à mesure que la société française, confronté à un terrorisme international relayé par des ramifications internes, voyait se profiler le spectre de l'anomie. Cette inquiétude s'est trouvée renforcée encore par le fait que tout un discours public a voulu établir une relation de continuité entre l'affirmation de l'identité et le développement de la radicalité.

\section{Le renouvellement des discours}

Le débat public s'est structuré, au cours de ces dernières décennies, autour de deux grandes polarités, «multiculturaliste » d'un côté, «universaliste» de l'autre, se plaçant du reste l'une et l'autre sous l'égide de l'idée de République. L'école "multiculturaliste», attachée à une laïcité inclusive, était encore dominante à l'aube des années 1990; elle s'est vue supplantée par l'école « universaliste» à partir du tournant du siècle. ${ }^{6}$

Porté par des figures comme celles d'Alain Touraine, de Michel Wieviorka, et d'Alain Renaut, le courant «multiculturaliste» se refuse à identifier la neutralité politique avec l'abstraction religieuse: un Etat est d'autant plus neutre

6 Nous avons présenté ce débat dans L'Etat et les religions en France (Portier, 2016a, chap. 9). 
qu'il accepte la pluralité en sa sphère même, sous certaines conditions. La neutralisation par abstention conduit à faire triompher la position majoritaire, qui n'a rien d'universel en soi. Pour les tenants de cette thèse, il ne s'agit pas de promouvoir les droits collectifs ou communautaires: cette option leur semble de nature, en effet, à pouvoir priver le sujet de sa capacité d'autodétermination. Le dessein est ici, bien plutôt, d'obtenir une reconnaissance du droit individuel à la différence: à la politique de l'égalité, aveugle devant la diversité des conditions, il faut substituer une politique de l'équité, acceptant le traitement différencié des sujets. Deux raisons justifient cette ouverture, qui tranche avec les réquisits autoritaires de l'"esprit jacobin ». D'une part, il est dans l'ordre de la démocratie constitutionnelle que chacun puisse exprimer librement ce qu'il ressent comme étant son "authenticité », pourvu que celle-ci, dans sa démonstration externe, ne remette pas en cause les droits d'autrui et l'ordre public. D'autre part, ajoutent les auteurs, en laissant les individus manifester extérieurement leur identité, la République favorise en fait leur intégration dans la société globale. Une redéfinition de la politique de laïcité accompagne ce discours de l'équité: elle doit évoluer dans le sens de la publicisation des identités. Cette ligne juridique, qui bouscule la séparation privé/public, n'est rien d’autre, d'ailleurs, qu'un prolongement des principes de la loi de 1905, dont l'orientation libérale, voulue par Briand, portait en germes déjà cette possibilité recognitive.7

L'école de la "laïcité universaliste» - qu'animent, dès la fin des années 1980, Catherine Kintzler, Henri Pena Ruiz, Elisabeth Badinter, aujourd'hui rejoints par Caroline Fourest ou Mohamed Sifaoui - entend bien, quant à elle, fixer dans le marbre la séparation traditionnelle du privé et du public, non cependant sans donner du "public» une définition bien plus extensive que ne le faisait la Troisième République: ici, l'impératif de neutralité suppose l'effacement du religieux, son "excommunication politique» (Ferry, 2016). Les arguments s'opposent trait pour trait à ceux de la ligne précédente. D'abord, l'expression des différences peut mettre en péril la liberté individuelle. On l'a vu avec le port du voile dans le domaine de l'école, que ces républicains veulent ériger en «sanctuaire». Il exprime l'abdication de soi, non seulement parce qu'il est souvent imposé par les proches, mais parce que per se, dans sa signification

7 Selon l'une des thèses de Jean Baubérot (2015). 
le plus profonde, il traduit la soumission à un ordre hétéronome où les femmes sont nécessairement, par un effet de la loi de Dieu, établies dans l'infériorité. On retrouve là, traduite dans la culture française de l'unité, la conception de la liberté républicaine comme non-domination. Dès 1989, Elisabeth Badinter et Régis Debray affirment que «tolérer le foulard islamique, ce n'est pas accueillir un être libre». Alain Finkielkraut ajoute au même moment qu'il faut faire la «distinction entre les droits de l'homme et les droits des tribus.$^{8}$ Ensuite, le multiculturalisme porte atteinte à la cohésion de la nation. C'est le grand thème du "communautarisme»: en autorisant la manifestation des identités singulières, l'Etat contribue à figer les individus dans des solidarités partielles qui les éloignent de la communauté globale. Catherine Kintzler (2015) exprimaitce point de vue, il y a peu encore:

La laïcité adjectivée (laïcité "plurielle», "ouverte», "positive», «raisonnable», "apaisée») récuse le caractère neutre et minimaliste de la puissance publique républicaine, faisant de l'opinion religieuse une norme, autorisant les propos religieux au sein de l'État lui-même, et aboutissant à légitimer la communautarisation religieuse du corps politique.

Il importe de préciser cependant que cette politique de l'homogène articule deux variantes: l'une, autour de Catherine Kintzler, rejointe récemment par des éditorialistes comme Natacha Polony ou des juristes comme Frédérique de la Morena, en tient pour une philosophie de la «raison abstraite», qui appelle le corps politique à se construire dans l'arrachement à toute tradition préalable; l'autre, autour d'Alain Finkielkraut, bientôt appuyé par des publicistes comme Eric Zemmour, fait valoir une philosophie de la "culture nationale», qui invite à enraciner la vie publique dans la mémoire «judéo-chrétienne». Si les deux variantes se défient des affirmations musulmanes, la seconde se montre bien plus accueillante que la première à la présence symbolique (mais non nécessairement normative ${ }^{9}$ ) des religions chrétienne et juive dans l'espace d'Etat, en ce qu'« elles ont fait la nation». Ensemble, ces deux variantes développent

8 Voir, sur ces points, Karel Leyva (2015).

9 Olivier Roy (2016, p. 46) le dit ainsi : «La continuité revendiquée entre christianisme et sécularisme moderne s'exprime aujourd'hui plus en termes d'identité que de valeurs.» 
une laïcité néo-gallicane: elles demandent à l'Etat de contraindre l'islam à s'inscrire dans le cadre des valeurs communes.

La sphère politique affiche les mêmes divisions que la sphère intellectuelle. On trouve en son sein une polarité inclusive. A gauche, elle a été portée par Lionel Jospin au début des années 1990, par Bernard Cazeneuve au cours de la période récente. A droite, par Edouard Balladur qui, en 1994, alors qu'il est Premier ministre, déclarait au journal Le Monde que «l'égalité, c'est le respect de l'identité». Alain Juppé a défendu également, au cours de ces dernières années, une "politique de la tolérance». Ce courant est généralement en phase avec l'approche de l'Observatoire national de la laïcité, annoncé par Jacques Chirac en 2003, et installé par François Hollande en 2013. Les attentats de ces dernières années ont cependant infléchi cette sensibilité: plus que dans les années 1990, qui se situaient encore, par certains traits, dans l'orbe du différencialisme de l'après-mai 1968, ses tenants insistent désormais sur la nécessité de faire droit aussi aux "valeurs de la République», envisagées cependant dans une perspective fort libérale. Peu à peu, ce courant, dont le propre est de défendre une laïcité "recognitive ${ }^{10}{ }^{10}$ vu son influence décliner, plus à droite qu'à gauche il est vrai. ${ }^{11}$

L'autre mouvance, qui a rencontré bientôt les faveurs de l'opinion publique, a entrepris de promouvoir une laïcité «identitaire», centrée principalement contre les affirmations musulmanes. Elle s'est construite à droite de la scène politique, mais a impliqué aussi une partie de la gauche autour, par exemple, de Manuel Valls ou Jean Glavany, ${ }^{12}$ eux-mêmes très soutenus par des associations laïques comme Egale ou des organes d'information militante comme Respublica. La contribution de la droite en la matière mérite un signalement particulier. En France, la laïcité est originellement une valeur de gauche. Liée au catholicisme, la droite a, dans un premier temps, résisté à sa consécration

10 Cette laïcité se distingue de la laïcité séparatiste (puisqu'elle permet un soutien de l'Etat aux Eglises ou du moins aux croyants) et de la laïcité gallicane (puisqu'elle admet la liberté d'organisation des groupements religieux).

11 Voir, par exemple, les productions de la Revue socialiste sur la laïcité (mars 2015) ou sur la République (décembre 2016).

12 Dans un communiqué rédigé avec Patrick Kessel et Françoise Laborde après le vote de la «loi travail» en juillet 2016, loi travail qui pourrait réduire la sphère de la liberté religieuse dans les entreprises, Jean Glavany déclare par exemple: «L'obsession de la garantie de la liberté ne doit pas entraîner la faiblesse dans la lutte contre les intégrismes.» 
institutionnelle, avant finalement, à partir des années 1920, de l'accepter dès lors qu'elle était appréhendée comme un régime de liberté religieuse. Reste qu'elle ne l'avait jamais mise au centre de son projet de société. Or, les choses se sont modifiées à la faveur de l'affaire du voile. Au début des années 1990, Charles Pasqua, ministre de l'Intérieur, lui consacre plusieurs interventions, comme du reste François Bayrou, ministre de l'Education nationale, lorsqu'en septembre 1994, il publie une circulaire visant à prohiber le foulard à l'école publique. Mais le texte principal en la matière relève de la littérature grise. Il s'agit du rapport que François Baroin, alors député, remet à Jean-Pierre Raffarin en juin 2003. Dans ce texte, intitulé significativement Pour une nouvelle laïcité, François Baroin (2016) entend "opérer une "repolitisation" du thème de la laïcité qui pourrait devenir une valeur de la "droite de mai" ».

François Baroin pose un diagnostic tout d’abord: la société françaiseconnaît une crise d'identité, du fait essentiellement de l'accueil trop favorable réservée (notamment par la gauche) aux revendications des populations immigrées d'origine musulmane:

Alors que notre société est bousculée par la mondialisation, la construction européenne et la décentralisation, la laïcité apparaît comme une référence stable et un peu mythique. C'est pourquoi elle est devenue un élément de référence de l'identité française. Sa remise en cause par le multiculturalisme et le communautarisme peut donc être perçue comme une menace pour l'identité nationale.

Le voile retient encore l'attention:

L'erreur de 1989 a été de considérer que le voile islamique était un signe d’appartenance religieuse alors qu'il s'agit en fait d'un attribut des fondamentalistes qui s'inscrit dans un modèle de société fondé sur une logique de ghetto et hostile aux valeurs de la démocratie.

On relèvera que le concept d'identité change alors de support. On l'employait dans les années 1960, avec Erikson et Goffmann, pour exprimer la singularité du sujet. Il prend désormais une valence collective: on s'y réfère pour évoquer la substance pérenne de la collectivité nationale. Comment inverser cette pente anomique? Le député de l'Aube dessine alors tout un programme d'intégration 
républicaine, articulé autour de l' "amélioration de la définition de la laïcité» (il s'agit là de contrer la jurisprudence du Conseil d'Etat), de la réaffirmation de la neutralité de l'Etat et de ses agents et de la redécouverte de l'éducation morale et civique. L'une des préconisations essentielles, assise sur l'idée qu' «à un certain point, les droits de l'homme et la laïcité peuvent être contradictoires », consiste à "proscrire le port du voile dans les établissements scolaires». Parallèlement, l'auteur reconnaît l'«apport des religions à notre culture» (il insiste sur les «racines chrétiennes » de la France), ce qui l'entraîne à militer en faveur de la création d'un Conseil consultatif des religions de France et d'une fiscalité plus favorable pour les institutions du culte («don spiritualité»).

Elu en mai 2007, le Président Nicolas Sarkozy reprend, en l'accentuant, la ligne dessinée par François Baroin: la réflexion du nouveau Président de la République articule également une ouverture "positive" à la croyance religieuse qu'il estime nécessaire à la constitution de l'éthique et de la mémoire communes de la société et une défiance à l'égard des conduites entropiques d'un islam qu'il faut replacer dans les cadres axiologiques de l'«identité nationale». On assiste de là - sous la pression, il est vrai, d'un Front national qui, après avoir longtemps donné dans la nostalgie de l'Etat catholique, s'est converti lui aussi à la laïcité identitaire - à une manière de «culturalisation » de la laïcité, enrôlée désormais davantage au service de la protection des «valeurs de la nation» que de la promotion des «libertés du sujet». On ne verra pas dans cette recomposition axiologique un produit exclusif de la pensée de droite. Il faut rappeler que la gauche est allée dans ce sens aussi, comme on le voit dans cette affirmation en 2010 du vice-président du Grand Orient de France devant la Commission d'enquête présidée par André Gerin concernant le port du voile intégral: «Dans l'espace public, la liberté individuelle doit s'exprimer dans les limites culturelles de la communauté nationale à une période donnée.»

\section{La réorganisation des règles}

Destinéà répondre au déficit de cohésion de la société contemporaine, ce nouveau cadre cognitif, articulé sur une double référence à la culture chrétienne et à l'humanisme rationaliste, a eu un effet reconfigurateur sur l'agencement du modèle français de laïcité. Produit de la victoire de la laïcité libérale d’Aristide Briand 
sur la laïcité gallicane d'Emile Combes, la loi de 1905 s'est construite sur le fondement d'une séparation stricte du privé et du public. Dans le privé, dont le concept intègre aussi l'espace de la société civile, l'expression du religieux est libre, sans autres contraintes que celles qu'imposent aux croyants les droits d'autrui et les exigences de l'ordre public. Réduite au monde de l'Etat et de ses fonctionnaires, la sphère publique doit être absolument neutre en revanche. Les interactions qui existent entre les Eglises et l'Etat, en matière par exemple de financement des édifices du culte dont la collectivité étatique est propriétaire, sont considérés comme de simples exceptions à la règle séparatiste. Il n'en va plus de même aujourd'hui. La dissociation fondatrice public/privé s'est trouvée, dans le droit même, largement atténuée. A sa place, s'est imposé un autre modèle de relation - d'intrication celui-là - entre la raison et la croyance, selon un double processus : l'Etat a voulu tout à la fois consolider la reconnaissance du religieux et renforcer sa surveillance. Cette politique de l'hybridité ne s'est pas construite sur l'assise de l'abrogation de la loi de 1905, mais sur le fondement de l'adjonction à ses dispositions initiales de législations, de réglementations et de jurisprudences nouvelles.

Le schéma de la reconnaissance s'était affirmée dans les années 1960-1970, porté tout à la fois par le dessein catholique du général de Gaulle et, bientôt, par la montée en puissance du différencialisme éthique. Il n'a pas été remisé depuis lors. Il a donné lieu, d'une part, à des politiques distributives. En contradiction avec ses règles premières (loi Goblet de 1886, loi de séparation de 1905), la République s'est engagée, depuis 1959, dans un financement massif des écoles privées, tout en respectant leur "caractère propre»; elle n'a pas hésité même à accorder des garanties d'emprunt et des subventions indirectes aux associations cultuelles, ou aux associations ordinaires qui entreprenaient de construire des édifices religieux. Le Conseil d'Etat a consacré, du reste, cette ouverture dans une série d'arrêts d'Assemblée du 19 juillet 2011, dès lors qu'un intérêt local est en jeu, et, selon la "théorie de l'objet mixte", que le financement ne vise pas à subventionner une activité exclusivement cultuelle. Il faut ajouter, d'autre part, les politiques symboliques. Certes, les autorités gouvernementales n'ont cessé, depuis les années 1990, de rappeler la neutralité de l'Etat, de ses espaces et de ses personnels.

Il reste que bien des "accommodements" ont été introduits au cours de la période récente. On admet, par exemple, que des autorisations d’absence 
puissent être accordées aux fonctionnaires lors des grandes fêtes de leur religion, que les cimetières puissent accueillir des carrés confessionnels, que, dans les hôpitaux, les «besoins spirituels» des patients puissent être intégrés dans les dispositifs de soins (ce qui excède la seule liberté de culte prévue par la loi de 1905). L'ouverture des programmes scolaires à l'enseignement des faits religieux manifeste une évolution dans le même sens, d'autant que l'Etat la justifie, non point seulement par des raisons cognitives (le comblement d'un déficit culturel), mais aussi par l'urgence, dans une société marquée par l'«accélération de la pluralisation", de mieux connaître l'autre dans sa différence culturelle et religieuse. En outre, au sein des administrations, se développent parfois des "zones grises", où se cristallisent, praeter legem, des pratiques subtiles de négociation avec la croyance: on a pu relever de la sorte que le voilement des cheveux était parfois toléré, pour les personnels mêmes de santé, dans certains services hospitaliers (Bertossi, 2015). Si l'Etat soutient les communautés confessionnelles, il attend d'elles aussi qu'elles le soutiennent. Il leur demande, par exemple, d'assumer des fonctions d'expertise (comme dans le cadre du Comité national d'éthique, au moins jusqu'à la présidence de François Hollande), ou des fonctions de médiation (comme dans les opérations de dialogue inter-religieux ou inter-convictionnel qu'il encourage), ou des fonctions de représentation (ce qu'assure, pour les musulmans, le Conseil français du culte musulman, établi sous l'égide de Nicolas Sarkozy en 2003). Ce qui vaut au niveau national vaut plus également au niveau local: il est fréquent, on le voit dans les diocèses de Versailles, de Créteil, de Clermont-Ferrand, que les élus, de la France Insoumise jusqu'au Front national, se retrouvent dans les arènes de dialogue mises en place par les évêques. Cette évolution générale répond à la dynamique même de la démocratie libérale: son souci de faire droit à l'«égale dignité» de ses assujettis l'a d’abord amenée à reconnaître leurs droits civils et civiques et, bientôt, sociaux; elle a accompagné ensuite, de plus en plus volontiers, leurs revendications sur le terrain des droits culturels, d'autant que la mondialisation juridique va clairement, depuis les années 1950, dans le sens de cette reconnaissance. Mais elle est un effet aussi de la crise du politique: confronté à son impotence matérielle et symbolique, l'Etat a besoin désormais, dans une société de plus en plus mobile et incertaine, de l'apport des ressources cognitives et matérielles des Eglises (Habermas, 2008a, 2008b, p. 7 s, 2008c). 
On touche là au second aspect de la «nouvelle laïcité». Peu évoqué dans les années 1960-1970, le souci de la cohésion - non seulement sociale mais aussi morale - s'est manifesté puissamment dans les années 1990-2000, comme en témoignent les rapports (Fragonnard, Delevoye, Debré, Stasi, Rossinot...) qui se sont succédé au cours de la période. Les attentats n'ont fait qu'amplifier ce désir de lien. L'idée n'est pas simplement d'amener les citoyens à respecter extérieurement les droits d'autrui et les principes constitutionnels qui les garantissent (ce que thématise le «devoir de civilité » propre au libéralisme traditionnel ${ }^{13}$ ), mais de les faire adhérer intimement aux «valeurs républicaines», et au "mode de vie» qu'on leur associe: à la limitation juridico-politique de la liberté de choix (qui se satisfait de réguler les comportements) s'ajoute donc sa limitation civicoculturelle (qui entend, en partie, configurer les convictions) (Joppke, 2010). Parce que le républicanisme, même dans sa version briandiste, excède en substance le simple libéralisme (en tant qu'il insiste sur l'intérêt commun et sollicite la «vertu» du sujet), cette tendance existait sans doute dans la France de la Troisième République. Elle s'est considérablement renforcée au cours de la période récente. La "politique transformative", à laquelle donne lieu ce programme intégrationniste, comporte deux volets. Un volet éducatif, d'une part, qu'illustrent l'affichage des "chartes de la laïcité» dans les administrations d'Etat, dans la fonction publique hospitalière, et, bien sûr, dans les écoles, mais aussi la réactivation de l'enseignement moral et civique dans les programmes scolaires. Un volet coercitif, d'autre part. Il faut faire arrêt ici sur les lois de 2004 sur le port des signes religieux ostensibles à l'école publique et de 2010 sur la dissimulation du visage dans l'espace public. Elles introduisent deux modifications essentielles dans le droit de la laïcité, qu'on trouverait de même dans les propositions de loi visant à proscrire le port des signes religieux à l'Université ou dans la loi «travail» de juillet 2016. ${ }^{14}$

13 John Rawls (1993).

14 Sur ce point, Philippe Portier (2016b, p. 61 s). A propos de la loi travail de juillet 2016, elle introduit en effet une rupture avec la législation antérieure, en affirmant dans son article ler que «le règlement intérieur [d'une entreprise] peut contenir des dispositions inscrivant le principe de neutralité et restreignant la manifestation des convictions des salariés si ces restrictions sont justifiées par l'exercice d'autres libertés et droits fondamentaux ou par les nécessités du bon fonctionnement de l'entreprise et si elles sont proportionnées au but recherché». 
Le législateur a redéfini les espaces d'application de la règle de la neutralité. Dans le modèle de 1905, comme on l'a souligné, seuls les espaces d'Etat étaient concernés par l'abstention religieuse. Encore s'agissait-il de l'imposer aux fonctionnaires simplement, et encore dans l'exercice de leur mission. Les deux lois à l'instant citées déplacent les frontières. Celle de mars 2004 étend aux usagers du service public de l'éducation, les élèves dans le primaire et le secondaire, une proscription que le Conseil d'Etat n'avait pas retenue dans son avis de novembre 1989 (sur le port du voile à l'école publique), non plus que dans sa jurisprudence subséquente. Celle d'octobre 2010, qui ne fait pas référence en tant que telle à la laïcité, va plus loin en prohibant de facto certains vêtements religieux pour les personnes ordinaires dans l'espace même du commun - la voie publique, les commerces ou les salles de spectacle - ce que la loi appelle, de manière inédite, l'«espace public». Le législateur a de surcroît réévalué les motifs de restriction de l'autonomie. La notion d'ordre public, qu'évoquent les textes de droit lorsqu'ils veulent limiter la liberté, et notamment la liberté religieuse, est ici centrale. Les autorités politiques (le législateur et le juge) l'avaient investie originellement d'une signification matérielle, en la renvoyant à des éléments objectifs (la sécurité, la tranquillité et la salubrité). Il la dote désormais d’une valence complémentaire, immatérielle celle-là, en la rapportant de plus en plus à un modèle substantiel de comportement, lié, selon l'expression du Conseil constitutionnel dans sa décision du 7 octobre 2010, aux «exigences minimales de la vie en société», ou, selon l'expression de la Cour européenne des droits de l'homme dans sa décision SAS c. France du ler juillet 2014, aux «conditions du vivre ensemble».

Dans la pratique, ces restrictions concernent l'islam, et non les autres confessions. Malgré quelques effets de halo sur les Eglises chrétiennes, les autorités publiques, comme on l'a déjà signalé, n'hésitent pas, même, à faire référence, mais le plus souvent en l'appariant à l'ordre démo-libéral, aux «racines chrétiennes de la France», et à publiciser, dans l'espace qu'ils régissent, certains symboles de la tradition comme les crèches de la nativité. Quelques analystes ont pu, de là, repérer l'existence, dans le droit français des cultes (mais la tendance est européenne), d'un «double standard» distinguant les droits pléniers des fidèles de la religion chrétienne et les droits plus limités des fidèles de la religion musulmane (Ferrari, 2012).

Le parcours des trente dernières années nous aura donc confrontés à un changement décisif dans la pratique de la laïcité. On peut décrire la mutation 
actuellement en cours comme le produit d'une réarticulation du rapport entre la norme et la valeur. La laïcité relevait, au début du XXe siècle, du registre de la norme: elle s'employait à permettre à chacun d'être traité, de manière juste, en tant que "personne libre et égale à toute autre» (Ferry, 2002). L'«intégrisme républicain" aurait aimé que l'Etat laïque puisse reconfigurer les religions, et même les éradiquer, dans le dessein d'accéder, au plus vite, le règne de la raison. Il ne l'a pas emporté. C'est, sous l'influence de Briand et Jaurès, le républicanisme libéral qui s'est imposé: le régime des cultes a pris la forme, en définitive, d'un cadre procédural permettant à chacun de cultiver sa croyance ou son incroyance à son gré, sans que l'Etat puisse peser directement sur l'organisation et l'activité des institutions religieuses. ${ }^{15}$ Cent-dix ans plus tard, on use autrement de la laïcité. Les gouvernements, de droite mais aussi de gauche (en tout cas, jusqu'à la présidence d'Emmanuel Macron, qui semble s'inscriredans une ligne plus inclusive, bien que non multiculturaliste), en ont fait un instrument de reconfiguration de l'esprit public: elle s'agence désormais en un dispositif de diffusion de la valeur, au service d'une politique du bien, et non point comme hier d'une politique du juste. C'est là le paradoxe de ce perfectionnisme libéral: il attente à la liberté religieuse, qui fait fond originellement sur la reconnaissance de l'autonomie de la personne, au nom du principe même d'autonomie.

\section{Références}

1972-2014: comment les Français ont évolué sur l'IVG. Atlantico, 12 févr. 2014. Disponible sur: <http://www.atlantico.fr/rdv/politico-scanner/1972-2014-comment-francais-ont-evolue-ivg-sonfage-ifop-978807.html>. Accès: 20 sept. 2017.

BAROIN, F. Pour une nouvelle laïcité. Paris, juin 2016. Rapport au Premier ministre. Club Initiative et Dialogue.

BAUBÉROT, J. Les sept laïcités. Paris: Maison de Sciences de l'homme, 2015.

BERGER, P. (Ed.). The desecularization of the world: resurgent religion and world politics. Grand Rapids: Eerdmans, 1999.

15 Ce qui n'empêchait pas, on l'a dit plus haut, que l'école puisse avoir un rôle recteur dans la formation de la conscience des élèves, dans le cadre cependant d'un régime qui laissait l'expression de la croyance religieuse en dehors de l'emprise de la sphère d'Etat. 
BERTOSSI, C. La citoyenneté à la française: valeurs et réalités. Paris: CNRS Éditions, 2015.

BRÉCHON, P.; GONTHIER, F. Les valeurs des européens: evolutions et clivages. Paris: Armand Colin, 2014.

CAMPBELL, J. E. Polarized: making sense of a divided America. Princeton: Princeton University Press, 2016.

FERRARI, A. Religious freedom and the public-private divide: a broken promise for Europe?. In: FERRARI, S.; PASTORELLI, S. (Ed.). Religion in public spaces: a European perspective. Ashgate: Farnham, 2012. p. 71-91.

FERRY, J.-M. Valeurs et normes: la question de l'éthique. Bruxelles: Éditions de l'Université de Bruxelles, 2002.

FERRY, J.-M. La raison et la foi. Paris: Pocket, 2016.

GALLAND, O.; MUXEL, A. Les jeunes et la radicalité religieuse et politique: une enquête auprès des lycéens. Rapport Gemass/Cevipof. Paris, 2017.

HABERMAS, J. De l'éthique de la discussion. Paris: Cerf, 1992.

HABERMAS, J. Les fondements prépolitiques de l'État de droit démocratique. In: HABERMAS, J. Entre naturalisme et religion: les défis de la démocratie. Paris: Gallimard, 2008a. p. 152-169.

HABERMAS, J. Qu'est-ce qu'une société “post-séculière”?. Le Débat, n. 152, p. 4-15, nov./déc. 2008b.

HABERMAS, J. Religion et sphère publique. In: HABERMAS, J. Entre naturalisme et religion: les défis de la démocratie. Paris: Gallimard, 2008c. p. 170-211.

HEGEL, F. Principes de philosophie du droit. Paris: Presses Universitaires de France, 1998.

JOPPKE, C. Citizenship and immigration. Cambridge: Polity Press, 2010.

KINTZLER, C. Femmes et laïcité. Paris, 19 mars 2015. Audition au Sénat. (Rapport sénatorial de la Délégation aux droits des femmes). Disponible sur: <http://www. senat.fr/compte-rendu-commissions/20150316/femmes.html\#toc2>. Accès: 20 sept. 2017.

LEYVA, K. Multiculturalisme et laïcité en France: les trois républicanismes du rapport Stasi. Dialogue: Canadian Philosophical Review, v. 54, n. 4, p. 647-684, Dec. 2015.

PORTIER, P. L'Etat et les religions en France: une sociologie historique de la laïcité. Rennes: PUR, 2016a. 
PORTIER, P. La politique du voile en France. Droits et valeurs dans la fabrique de la laïcité. Revue du droit des religions, n. 2, p. 79-81, nov. 2016b.

RAWLS, J. Libéralisme politique. Paris: Presses Universitaires de France, 1993.

ROY, O. La laïcité à l'épreuve de l'islam. Paris: Stock, 2005.

ROY, O. Pour des sociétés ouvertes. Repenser la place des religions en Europe. Esprit, n. 422, p. 44-58, févr. 2016.

WILLAIME, J.-P. Reconfigurations ultramodernes. Esprit, n. 3, p. 146-155, mars/avril 2007.

Recebido: 30/09/2017 Aceito: 11/05/2018 | Received: 9/30/2017 Accepted:5/11/2018 